\title{
Scaling of genuine multiparticle entanglement close to a quantum phase transition
}

\author{
Martin Hofmann, ${ }^{1}$ Andreas Osterloh, ${ }^{2}$ and Otfried Gühne ${ }^{1}$ \\ ${ }^{1}$ Naturwissenschaftlich-Technische Fakultät, Universität Siegen, Walter-Flex-Str. 3, 57068 Siegen, Germany \\ ${ }^{2}$ Fakultät für Physik, Campus Duisburg, Universität Duisburg-Essen, Lotharstr. 1, 47048 Duisburg, Germany
}

(Dated: September 19, 2018)

\begin{abstract}
We investigate the scaling and spatial distribution of genuine multiparticle entanglement in threeand four-spin reduced states of the one-dimensional XY-model at the quantum phase transition. We observe a logarithmic divergence, show that genuine three- and four-particle entanglement obeys finite-size scaling of the XY-model and demonstrate that the genuine three-particle entanglement has a finite spatial range.

PACS numbers: 03.65.Ud, 03.67.Mn
\end{abstract}

\section{INTRODUCTION}

In recent years it has turned out that quantum correlations play an important role in several branches of physics. One of these branches is the field of quantum optics and, more specifically, quantum information processing, where quantum correlations are often a useful resource for tasks like quantum-enhanced precision measurements or quantum key distribution. Another branch is the field of condensed matter physics: here, the study of entanglement in ground and thermal states of spin models has shed some light on the nature of quantum phase transitions [1 [6], and in the same time has provided new insights into the simulation of spin models [7].

Most of the existing studies of entanglement in spin models, however, have restricted their attention to bipartite entanglement (for exemptions see [5, 8 [1] ]): Either, the entanglement in the reduced state of two particles of a multiparticle system was studied [1, 2], or the entanglement between one block of particles and the remainder of the system [3]. In the latter case, the entanglement can often be related to the surface of the block, and these socalled area laws are an active research topic in mathematical physics [3, 13], for a review see [14]. The restriction to two-party entanglement is due to the fact that the theory of entanglement for multiparticle systems is still not fully developed, and many open problems exist. Therefore, in the existing works on multiparticle entanglement in spin models [9 11] only bounds on the entanglement are given. In a complementary approach Batle et al. 12 investigated nonlocality in spin chains using Mermin inequalities. The presence of nonlocality however, does not imply the presence of genuine multiparticle entanglement.

In this paper we study the scaling and spatial distribution of genuine multiparticle entanglement at a quantum phase transition in one-dimensional spin models. Our results are enabled by recent progress in the theory of multiparticle entanglement 15, 16] in combination with an explicit determination of reduced $k$-particle states in the transverse XY model [17]. We consider a computable measure for genuine multiparticle entanglement for the reduced three- and four-particle states and demonstrate that its derivative diverges at the critical point. For both cases we show that the entanglement obeys finite-size scaling, which can be used to compute the critical exponent for the infinite system from finite-size data.

\section{THE MODEL}

We consider the one-dimensional XY model with transverse magnetic field on $L$ particles and periodic boundary conditions [17]. The Hamiltonian of this model is given by

$H=-\sum_{i=1}^{L} \frac{\lambda}{4}\left[(1+\gamma) \sigma_{x}^{(i)} \sigma_{x}^{(i+1)}+(1-\gamma) \sigma_{y}^{(i)} \sigma_{y}^{(i+1)}\right]+\frac{1}{2} \sigma_{z}^{(i)}$,

where the coupling constant $\lambda \geq 0$ tunes the strength of the nearest neighbor coupling with respect to the external magnetic field. The parameter $\gamma$ sets the anisotropy of the system and connects the Ising model $(\gamma=1)$ with the isotropic XY model $(\gamma=0)$. In the thermodynamic limit and for $0<\gamma \leq 1$ the ground state of the model undergoes a quantum phase transition at the critical point $\lambda_{c}=1$. For $\lambda=0$ there is a unique ground state, where all spins are aligned in the direction of the magnetic field and there is no magnetization in the $X Y$-plane. For $\lambda \rightarrow \infty$ the ground state is two-fold degenerate and hence is an equal mixture of these two states. At the quantum phase transition the systems ground state changes from being non-degenerate to degenerate, accompanied by an abrupt change of the magnetization in the $x$-direction which is zero for $\lambda<1$ and finite for $\lambda \geq 1$. We use the $X Y$-model as a paradigm for our approach since it allows to study a phase transition with analytical rigor [17]. In the following, we focus on the transverse Ising model $(\gamma=1)$, but our approach can be straightforwardly extended to the $X Y$-model.

\section{GENUINE MULTIPARTICLE NEGATIVITY}

Our tool to study genuine multiparticle entanglement in the reduced marginals of the ground state of the 
spin model is the genuine multiparticle negativity [15]. This mixed state entanglement monotone vanishes on all biseparable states and a nonzero value is a sufficient criterion for genuine multiparticle entanglement to be present. Let us sketch its main idea and give the related definitions for three parties, Alice (A), Bob (B) and Charlie (C), the generalization to more parties is straightforward.

First, recall that a three-particle state is fully separable and contains no entanglement, if it can be written as a statistical mixture of product states, that is $\varrho^{\text {fulsep }}=\sum_{k} p_{k}\left|\psi_{A}^{k}\right\rangle\left\langle\psi_{A}^{k}|\otimes| \phi_{B}^{k}\right\rangle\left\langle\phi_{B}^{k}|\otimes| \phi_{C}^{k}\right\rangle\left\langle\phi_{C}^{k}\right|$. If the state is not fully separable, then it contains some entanglement, but it might be still separable with respect to one of the splittings $A|B C, B| A C$ or $C \mid A B$. In this case it can be written as statistical mixture of states on the first system and joint states on the last two systems, for example $\varrho_{A \mid B C}^{\text {sep }}=\sum_{k} p_{k}\left|\psi_{A}^{k}\right\rangle\left\langle\psi_{A}^{k}|\otimes| \phi_{B C}^{k}\right\rangle\left\langle\phi_{B C}^{k}\right|$. In this case, the state is called biseparable and not all three particles are entangled. Therefore, a state is called genuine multiparticle entangled [29] if it is not a mixture of biseparable states, that is, it can not be written as

$$
\varrho^{\text {bisep }}=p_{A} \varrho_{A \mid B C}^{\mathrm{sep}}+p_{B} \varrho_{B \mid A C}^{\mathrm{sep}}+p_{C} \varrho_{C \mid A C}^{\mathrm{sep}} .
$$

Clearly, for genuine multiparticle entangled states all particles are entangled and therefore this is the most interesting form of multiparticle entanglement 19.

In order to study genuine multiparticle entanglement, it was proposed in Ref. [15] to consider so-called PPT (positive partial transpose) mixtures. These are of the form

$$
\varrho^{\mathrm{PPTmix}}=p_{A} \varrho_{A \mid B C}^{\mathrm{PPT}}+p_{B} \varrho_{B \mid A C}^{\mathrm{PPT}}+p_{C} \varrho_{C \mid A C}^{\mathrm{PPT}},
$$

where $\varrho_{A \mid B C}^{\mathrm{PPT}}$ is a state for which the partial transpose with respect to the partition $A \mid B C$ has no negative eigenvalues [30]. Since separable states are also PPT [20], the set of PPT mixtures is a superset of the biseparable states and hence all states which are not PPT mixtures are genuine multiparticle entangled. The advantage of the approach of Ref. 15] is that the question whether a state is a PPT mixture or not can be decided directly, moreover, although PPT mixtures are only an approximation, the resulting entanglement criteria are the strongest criteria known. In fact, for many families of states (e.g. permutationally invariant three-qubit states), they are even necessary and sufficient for genuine multiparticle entanglement 21].

The genuine multiparticle negativity $N_{\varrho}$ is a measure to distinguish between states that are PPT mixtures and such that are not. It vanishes, if a decomposition into a PPT mixture exists and is nonzero elsewhere. Moreover, it was proven to be an entanglement monotone, since it cannot increase under local operations and classical communication [15]. At this point, we mention that it can be computed directly for a given density matrix via semidefinite programming, the precise formulation is given in the Appendix A1.

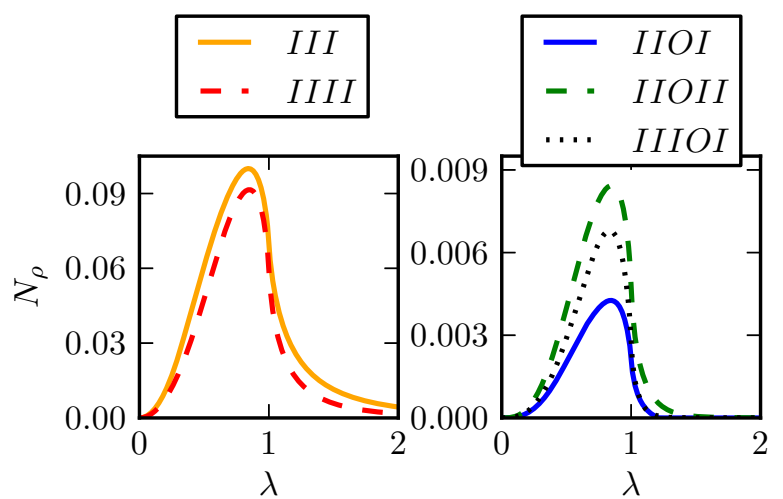

FIG. 1: The genuine negativity $N_{\rho}$ (for $L \rightarrow \infty$ ) as a function of $\lambda$ for three- and four-particle reduced states in different spatial arrangements. On the left the values for three (III) or four (IIII) consecutive particles are shown. On the right hand side, spatial arrangements for three and four particles which contain a single vacancy (denoted by 0 ) are shown. Here, II0I denotes a three-particle reduced state in the qubits $\{i, i+1, i+3\}$ and IIOII denotes a four-particle reduced state in the qubits $\{i, i+1, i+3, i+4\}$ etc. See text for further details.

\section{ENTANGLEMENT IN REDUCED THREE-QUBIT STATES}

Let us start with the three-particle marginals of the ground state of the Ising model. Consider the particles $i, j$ and $k$ in the systems ground state. For the Ising model we can always set $i$ to be zero, since the $X Y$ Hamiltonian is translationally invariant and has periodic boundary conditions. The spatial arrangement of the particles is then completely described by $(\alpha, \beta)=(j-$ $i, k-j)$, where $(\alpha, \beta)$ and $(\beta, \alpha)$ lead to the same reduced states due to mirror symmetry and hence one can choose $\alpha \leq \beta$ without loss of generality.

As a first task, we determined explicitly the reduced three-qubit states using the methods of Ref. [17], detailed expressions are given in the Appendices A5 to A7, Evaluating the genuine multiparticle negativity for different spatial constellations in the thermodynamic limit $L \rightarrow \infty$ we find that for $(1,1)$ and $(1,2)$ the reduced marginals of the ground state are genuine multiparticle entangled in the vicinity of $\lambda=1$ (see Fig. (1) and no entanglement is found if one separates the particles further. As the criterion of PPT mixtures is in general only a sufficient criterion for entanglement, the question arises whether for the separated configurations the reduced states are indeed biseparable. Concerning this point, it it first worth mentioning that so far no example of a genuinely entangled three-qubit state which cannot be detected by the PPT mixture approach is known. In our case, we can show even explicitly, using the algorithm for proving separability from Ref. [16] that the states are separable if the qubits are separated further (see Appendix A 2). Using these novel results we also conclude that the 
genuine three-particle entanglement in the ground state stays short ranged and falls off to zero. It answers a discussion recently raised in Ref. 9]: In this reference, lower bounds on the entanglement were computed and no entanglement in the configuration $(1,2)$ was found, so it remained open, whether this or other configurations were separable. This makes us confident that the genuine multiparticle negativity is a well-suited tool for our analysis.

\section{FINITE-SIZE SCALING}

The observed divergence indicates that the system undergoes a phase transition. We study it in more detail using the finite size scaling analysis [22, 23]. This analysis is based on the idea that close to the phase transition at $\lambda_{c}$ the behavior of a diverging quantity $P^{(L)}(\lambda)$ for finite system sizes $L$ is governed by the system size $L$ and a rescaled variable $L / \xi$ only, with $\xi$ being the correlation length. In case of a logarithmic singularity of $P^{(L)}(\lambda)$, the finite-size scaling ansatz asserts the existence of a function $Q$, such that for finite $L$ and $\lambda$ close to the critical value [23]

$$
P^{(L)}(\lambda)-P^{(L)}\left(\lambda_{0}\right) \sim Q\left(L^{\frac{1}{\nu}}\left|\lambda-\lambda_{c}\right|\right)-Q\left(L^{\frac{1}{\nu}}\left|\lambda_{0}-\lambda_{c}\right|\right) .
$$

Here $\nu$ is the critical exponent, which governs the divergence of the correlation length $\xi \sim\left|\lambda-\lambda_{c}\right|^{-\nu}$ close to the critical value as $L \rightarrow \infty$. For the ansatz to consistently recover

$$
P^{(\infty)}(\lambda) \sim C_{\infty} \ln \left|\lambda-\lambda_{c}\right| \text { as } \lambda \rightarrow \lambda_{c}
$$

in the thermodynamic limit, one sets $Q(z) \sim C_{\infty} \ln z$ for $z \rightarrow \infty$. Provided that $Q(z)=$ const. as $z \rightarrow 0$

$$
P^{(L)}\left(\lambda_{c}(L)\right) \sim-\frac{C_{\infty}}{\nu} \ln L+\text { const. }
$$

the minimum in $P^{(L)}$ at the pseudo-critical value diverges with the system size $L$, such that the equations (5) and (6) allow to determine the critical exponent $\nu$.

For the two-particle entanglement, it was already shown that finite-size scaling holds [1]. In the multiparticle case, the quantity of interest is the derivative of the multiparticle negativity. So we vary the particle number $L$, keeping the arrangement $(1,1)$ fixed, and study the three-particle genuine negativity $N_{\rho}^{(L)}$ together with its first derivative $\partial_{\lambda} N_{\rho}^{(L)}$ (see Fig. 2). One observes a logarithmic divergence of $\partial_{\lambda} N_{\rho}^{(\infty)}$ at $\lambda_{c}=1$. This is where the quantum phase transition occurs. There is distinct minimum in $\partial \lambda N_{\rho}^{(L)}$ for finite system sizes $L$ at $\lambda_{c}(L)$ which we take to be the pseudo-critical value. We find that it approaches the critical value like $\lambda_{c}(L)-\lambda_{c} \sim L^{-\kappa}$ with a shift exponent $\kappa=2.19$ 31.

Fitting the expected behavior to our data as done in Fig. 3. one recovers the critical exponent, which is known

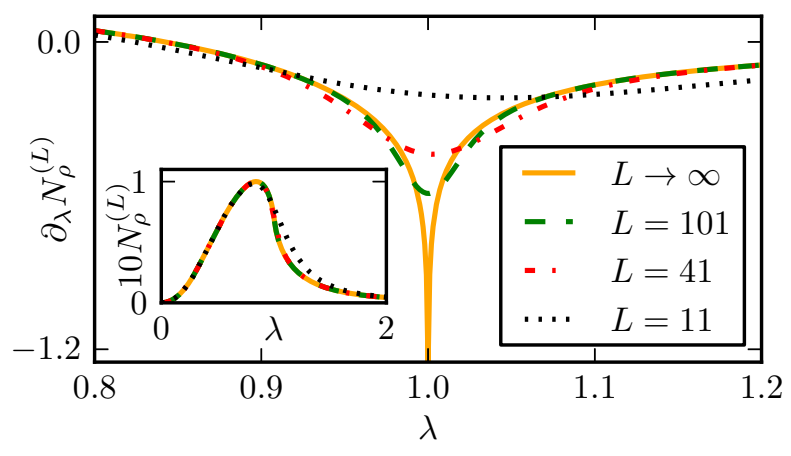

FIG. 2: In the thermodynamic limit the first derivative of the genuine negativity with respect to $\lambda$ diverges at the quantum phase transition. For finite chain length $L=11$ (dotted line), $L=41$ (dashed dotted line) and $L=101$ in (dashed line) there is a minimum in the vicinity of the critical point $\lambda_{c}=1$, which gets smaller for increasing $L$, diverging in the limit $L \rightarrow \infty$. The subplot shows the genuine negativity depending on $\lambda$ for different chain lengths in a larger region around the critical point.

to be $\nu=1$, since

$$
\begin{aligned}
\partial_{\lambda} N_{\varrho}^{(\infty)} & =0.170 \ln \left(\left|\lambda-\lambda_{c}\right|\right)+0.267 \\
\partial_{\lambda} N_{\varrho}^{(L)}\left(\lambda_{c}(L)\right) & =-0.170 \ln L+0.191,
\end{aligned}
$$

for sufficiently big $L$. We performed the same analysis on the genuine negativity for the arrangements $(1,2)$ and observed similar qualitative results. Note that the numerical accuracy of the fitting procedure is higher than the displayed accuracy, a more detailed discussion is given in Appendix A3

The finite-size scaling analysis shows that the behavior of the genuine multiparticle entanglement close to the critical point is governed by the quantum phase transition. A divergent behavior alone may be expected from the results of Ref. [4], as the reduced two-particle density matrix of the ground state itself is non-analytical [32]. Indeed many investigations of lower bounds on genuine multiparticle entanglement show this behaviour [9 11] . On the flip side non of these investigations allowed to draw conclusions with respect to the critical attributes of the system. The finite-size scaling, however, shows that multiparticle entanglement faithfully represents important properties of the spin system at the critical point, moreover, it may be used for the extrapolation of critical exponents from finite-size numerical simulations.

\section{ENTANGLEMENT IN REDUCED FOUR-QUBIT STATES}

As in the previous part, the density matrices of four particles $i<j<k<l$ depend on the spacing between the particles $\alpha=j-i, \beta=k-j$ and $\delta=l-k$ and the 


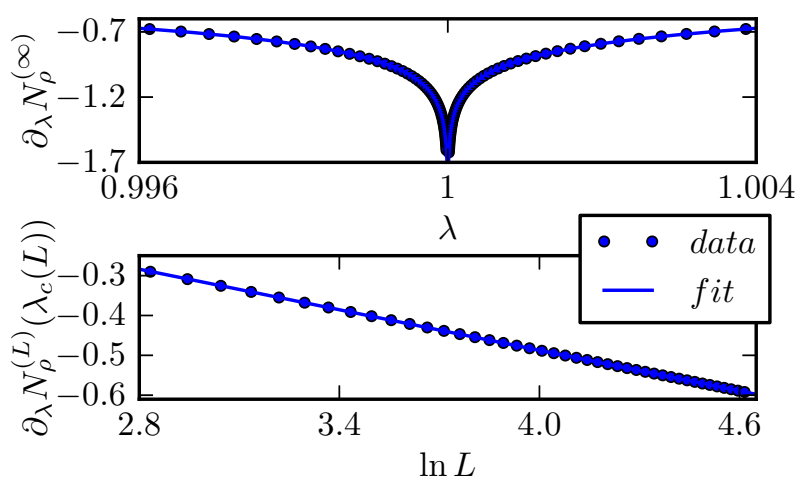

FIG. 3: Evaluation of the minima for different chain lengths and the divergence in the thermodynamic limit (circles) of the first derivative of the genuine negativity for three consecutive particles shows the behavior expected (lines) from the scaling ansatz for a logarithmic divergence. In the upper graph the divergence of the genuine negativity is plotted with respect to the coupling parameter $\lambda$ close to the critical point $\lambda_{c}=1$. The lower plot shows that minimum at the pseudo-critical value $\lambda_{c}(L)$ scales linear with the logarithm of the chain length $L$.

coupling parameter $\lambda$ only. Here we may choose $\alpha \leq \delta$ due to symmetry.

We find that there are three spatial arrangements with non-zero genuine negativity. These are the tightest packed constellations $(1,1,1)$ and the constellations $(1,1,2)$ and $(1,2,1)$. Further separated constellations yield a zero genuine negativity. We observe that with increasing separation the four-particle genuine negativity decreases with increasing separation. This is similar to the three particle case. On the other hand, the genuine negativity for the four-particle arrangements with one intermediate particle $(1,1,2)$ and $(1,2,1)$ is much larger than the comparable three-particle case $(1,2)$ (see Fig. 1). Taking into account that for the tightest conformations $(1,1)$ respectively $(1,1,1)$ the values are quite close to each other the four-particle genuine multiparticle entanglement seems to be more uniformly distributed throughout the system.

In addition, we tested the four-concurrence, defined on pure states as $C_{4}(\psi):=\left\langle\psi^{*}\left|\sigma_{y} \otimes \sigma_{y} \otimes \sigma_{u} \otimes \sigma_{y}\right| \psi\right\rangle$ and analytically extendable to mixed states [24] on this model, and it qualitatively behaves roughly the same way, except for single distribution patterns. Since $C_{4}$ is non-zero only for products of two particle Bell states and $G H Z_{4}$ type of states, this observation suggests two possible scenarios: either the state is a mixture of Bell products, not seen by $N_{\rho}$ plus states from the null-cone (for example $W_{4}$ states), or it really contains $G H Z_{4}$ type entanglement. Further studies in this direction are needed in order to clarify this issue.

The finite-size scaling analysis for four consecutive particles $(1,1,1)$ yields results similar to the three-particle case but is more subject to numerical errors due to error propagation. For separations $(1,2,1)$ and $(1,1,2)$ the first derivative of the genuine negativity shows a qualitatively similar scaling behavior as in the case where all four particles are in succession. A full discussion is given in the Appendix $\mathrm{A} 4$

\section{DISCUSSION}

Using the Ising model in a transverse magnetic field, we investigated the connection between genuine multiparticle entanglement and quantum phase transitions. We identified the configurations of three and four particles where entanglement is present and showed that the derivative of the genuine multiparticle negativity diverges logarithmically at the critical points. We further confirmed that this quantity obeys a finite-size scaling behavior close to the quantum phase transition.

Besides its fundamental interest, there are several consequences and applications of our work. First, as our method allows the direct study of multiparticle entanglement, it can be used to complete existing indirect results on multiparticle entanglement. To give an example, in Ref. [5] the existence of multiparticle entanglement in the one-dimensional XYZ model was concluded indirectly from monogamy relations, and possible connections to phase transitions were found. Our approach allows to verify these results in a direct manner. Similarly, in Ref. [8] the two-partite negativities for different splittings of three particles in the XY model were studied, our methods can now decide whether these bipartite quantities are connected to genuine multiparticle entanglement. Further, in Ref. [10] certain three-particle reduced could neither be detected as genuine multiparticle entangled nor shown to be biseparable. We expect that an application of our method would solve this issue. Second, our results demonstrate the usefulness of the genuine multiparticle negativity to study many-body systems. This makes it applicable to further systems, such as dynamical phase transitions [25], quenching dynamics, or the study of symmetry breaking [10].

\section{Acknowledgments}

We thank Steve Campbell, Tobias Moroder and Anna Sanpera for discussions. This work has been supported by the EU (Marie Curie CIG 293993/ENFOQI) and the BMBF (Chist-Era Project QUASAR). A.O. acknowledges the financial support by the SFB TR12. 


\section{Appendix A}

\section{Mathematical definition of the multiparticle negativity}

Mathematically, the genuine multiparticle negativity is defined as optimization over the set of fully decomposable witnesses $W$, these witnesses have to fulfill certain constraints [15]. In total, the optimization problem reads for three particles:

$$
\begin{aligned}
& N_{\varrho}=-\min _{W} \operatorname{tr}(W \varrho) \\
& \quad \text { such that for all } m \in\{A, B, C\} \\
& \quad W=P_{m}+Q_{m}^{T_{m}}, 0 \leq P_{m}, Q_{m} \leq \mathbb{1} .
\end{aligned}
$$

As all these conditions are linear or semidefinite constraints, this problem can be solved with the method of semidefinite programming, and the optimality of the solution can be verified. A simple, ready-to-use implementation can be found on-line [26].

\section{Separability of three and four qubits}

In our work we supplement the entanglement monotone with a separability algorithm [16]. The idea of the algorithm is to decompose $\varrho$ into two biseparable parts

$$
\varrho=(1-p) \varrho_{a}+p \varrho_{b},
$$

such that $\varrho_{b}$ is a statistical mixture of pure separable states and $\varrho_{a}$ is within the ball of separable states around the completely mixed state [27]. The algorithm is performed iteratively such that the purity of $\varrho_{a}$ is decreased with each successful step until $\varrho_{a}$ lies within the set of separable states proving the separability of $\varrho$.

Given an input state $\varrho$, we define $\varrho=\varrho_{0}$ and iterate the following procedure:

- Find a pure biseparable state $\left|\psi_{k}\right\rangle$ which has large overlap with $\sqrt{\varrho_{k}}$. It will add to the part $\varrho_{b}$ in the decomposition (A2). We take random states and choose the one with largest overlap.

- Choose $0 \leq \varepsilon_{k} \leq 1$, such that $\varrho_{k+1}=\frac{1}{1-\varepsilon_{k}}\left(\varrho_{k}-\varepsilon_{k}\left|\psi_{k}\right\rangle\left\langle\psi_{k}\right|\right)$ is positive semidefinite. This ensures, that $\varrho$ is indeed a convex combination of all $\left|\psi_{k}\right\rangle\left\langle\psi_{k}\right|$ and $\varrho_{k+1}$. Note that the algorithm seems to be more reliable, if $\varepsilon_{k}$ is significantly smaller than the smallest eigenvalue of $\varrho$.

- Check whether $\operatorname{tr}\left(\varrho_{k+1}{ }^{2}\right)<\frac{1}{7}$ and thus if $\varrho_{k+1}$ is separable for some bipartition [27].

- If this is the case, than the algorithm finishes and $\varrho$ is separable, else continue until some maximal iteration number is reached.

In practice the algorithm performs good on all full rank states, if however the state has eigenvalues close to zero it fails to detect separable states as such. This is due to the fact, that $\rho_{0}$ is initially close to the border of separable states and hence during iterations $\rho_{k}$ might leave the set of separable states. In such a case the algorithm can not bring back $\rho_{k}$ into the separable ball around the completely mixed state and the algorithm does not detect the state as separable.

Using local filtering one can significantly improve the algorithms ability to detect separable states with small eigenvalues. The idea is to apply invertible local matrices to the state $\varrho \mapsto \mathcal{F}_{1} \otimes \mathcal{F}_{2} \otimes \mathcal{F}_{3} \varrho \mathcal{F}_{1}^{\dagger} \otimes \mathcal{F}_{2}^{\dagger} \otimes \mathcal{F}_{3}^{\dagger}$, such that the smallest eigenvalue of the normalized new state increases. This new states is then more likely to be detected by the algorithm if it is separable. Since both states are connected by invertible local operations the separability of one state implies the separability of the other. Using this modification we can on the one hand decrease the number of iterations the algorithm needs to show the separability of a separable state. On the other hand we are able to detect separable states, which are not detected by the unmodified algorithm.

Our investigations of the three-particle reduced states in the thermodynamic limit for the particle separation other than $(1,1)$ and $(1,2)$ result in a vanishing genuine multiparticle negativity. Applying the algorithm to states of the constellations $(1,3)$ and $(2,2)$ confirms that these reduced three-particle marginals are separable (see Fig. (4). 

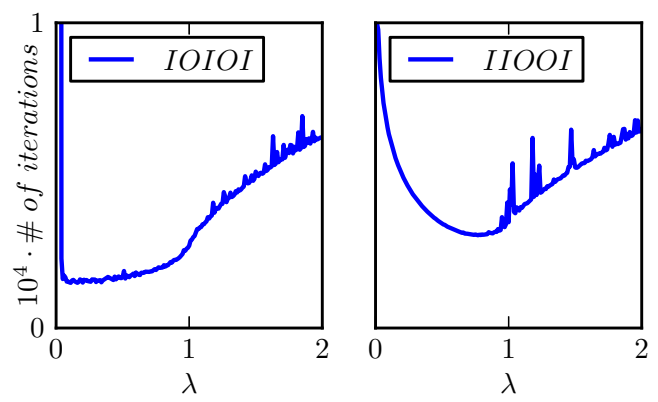

FIG. 4: The number of iteration steps of the separability algorithm needed to show separability is shown with respect to the coupling parameter $\lambda$ for the particle constellations $(1,3)$ (right) as well as for $(2,2)$ (left). For most cases the algorithm converges in less than $10^{4}$ steps proving the separability of the respective states. For $\lambda=0$ we know that the reduced state is pure and separable. For $\lambda \rightarrow 0$ the underlying state has small eigenvalues. Here the algorithm does not prove separability, even though these states are separable as well.

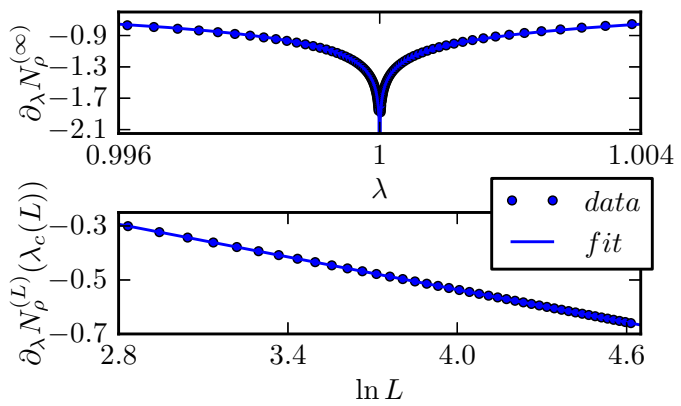

FIG. 5: Evaluation of the minima for different chain lengths and the divergence in the thermodynamic limit (circles) of the first derivative of the genuine negativity for four consecutive particles shows the scaling behavior expected (lines) from the scaling ansatz for a logarithmic divergence. On top the genuine negativity is plotted with respect to $\lambda$ diverging logarithmically at the critical point. The mid plot confirms the ansatz made with respect the $L$-dependence of position of the minima and the lower plot shows, the predicted behavior of the value of the minima with respect to different chain length.

\section{Four successive particles scaling analysis}

The scaling analysis in the case of four closely packed particles $(1,1,1)$ is along the line of the three-particle case. It yields similar results as in the three-particle case (see figure 5)

$$
\begin{aligned}
\partial_{\lambda} N_{\varrho}^{(\infty)} & =0.20 \ln \left(\left|\lambda-\lambda_{c}\right|\right)+0.36 \\
\partial_{\lambda} L_{\varrho}^{(L)}\left(\lambda_{c}(L)\right) & =-0.20 \ln L+0.27 .
\end{aligned}
$$

The negative quotient of the logarithmic prefactors again give way to the expected critical exponent of the diverging correlation length $\nu=1$. Due to numerical precision, however, the determination of the position and value of the minima was more subtle than in the tree particle case. Especially a faithful determination of the position of the minima was impossible for $L>33$ due to numerical inaccuracies, which we discuss in the next section in this appendix. The determination of the absolute value of the minima however suffered less from these problems and made it possible to confirm the expected scaling.

\section{Discussion of numerical precision}

For the derivation of our results we make use of the genuine negativity [15], which is implemented via semidefinite programming. It provides an upper bound of how far the numerical value obtained is from the global optimum. One 
can take this bound as a conservative error for the genuine negativity. In our calculations the absolute error of the genuine negativity is of the order of $10^{-14}$. This error is negligible, if one considers the genuine negativity itself. For the finite-size scaling analysis however this error has to be taken into account. Recall, that in the scaling analysis one has to extract several minima of the first derivative of the genuine negativity. The first derivative is approximated with the central finite difference method

$$
f^{\prime} \approx \frac{-f(x+2 h)+8 f(x+h)-8 f(x-h)+f(x-2 h)}{12 h}
$$

and the forward and backward finite differences close to the divergence

$$
\begin{aligned}
& f^{\prime} \approx \frac{-25 f(x)+48 f(x+h)-36 f(x+2 h)+16 f(x+3 h)-3 f(x+4 h)}{12 h} \\
& f^{\prime} \approx \frac{+25 f(x)-48 f(x-h)+36 f(x-2 h)-16 f(x-3 h)+3 f(x-4 h)}{12 h} .
\end{aligned}
$$

We found $h=10^{-7}$ to give us optimal results. The absolute error of the genuine negativity, results in an absolute leading error in the first derivative. It is of the order of $10^{-7}$ and hence the overall precision in estimating the position and absolute value of the minimum in the first derivative of the genuine negativity is limited. This directly influences how well one can perform the finite-size scaling analysis. In case of three $(1,1)$ and four $(1,1,1)$ consecutive particles one can find the position of these minima with sufficient high precision up to $L=33$ particles and their values up to $L=100$ particles, which is sufficient for the finite-size scaling analysis. For a larger number of particles however the errors of the positions and values of the minima caused growing errors in the coefficients of the fitting functions. This finally makes the extraction of the critical exponent $\nu$ impossible. Hence, we omitted a quantitative finite-size scaling analysis in these cases.

\section{Diagonalizing the XY model}

We give a brief review of the analytical diagonalization of

$$
H=-\sum_{i=1}^{L} \frac{\lambda}{4}\left[(1+\gamma) \sigma_{x}^{(i)} \sigma_{x}^{(i+1)}+(1-\gamma) \sigma_{y}^{(i)} \sigma_{y}^{(i+1)}\right]+\frac{1}{2} \sigma_{z}^{(i)}
$$

The strategy is as follows [17].

- First, we apply the so called Jordan-Wigner transformation, which maps the model onto a fermionic Fock space with creation and annihilation operators.

- Second, the transformed system decouples into a direct sum of four dimensional Hilbert spaces by using discrete Fourier transformation.

- Finally, these subspaces can be diagonalized, giving access to the ground state, the energy spectrum and expectation values of finite products of one site Pauli operators.

First, apply the Jordan-Wigner transformation, which transforms the spin operators $S_{\alpha}^{j}, j=1, \ldots, L, \alpha=x, y, z$ into fermionic creators $c_{j}^{\dagger}$ and annihilators $j$. It is composed of two intermediate transformations

$$
a_{j}^{\dagger}=S_{x}^{j}+i S_{y}^{j} \text { and } a_{j}=S_{x}^{j}-i S_{y}^{j}
$$

where $a_{j}^{\dagger}$ and $a_{j}$ are hard-core bosonic creation and annihilation operators. The fermionic operators are then obtained by

$$
\mathrm{j}=\exp \left\{\pi i \sum_{k=1}^{j-1} a_{k}^{\dagger} a_{k}\right\} a_{j}, \quad c_{j}^{\dagger}=a_{j}^{\dagger} \exp \left\{-\pi i \sum_{k=1}^{j-1} a_{k}^{\dagger} a_{k}\right\} .
$$

The new operators obey the fermionic anti-commutation algebra

$$
\left\{c_{i}^{\dagger}, \dot{j}\right\}=\delta_{i j} \text { and }\{\dot{i}, \dot{j}\}=\left\{c_{i}^{\dagger}, c_{j}^{\dagger}\right\}=0 \text {. }
$$


Applying the Jordan-Wigner transformation to the Hamiltonian (A7) yields

$$
H=\frac{L}{2}+\frac{\lambda}{2}\left[\left(c_{L}^{\dagger} \frac{1}{3}+\gamma c_{L}^{\dagger} \frac{1}{3}\right)+\text { h.c. }\right]\left(\exp \left\{i \pi \sum_{j=1}^{L} c_{j}^{\dagger} \dot{j}\right\}+1\right)-\frac{\lambda}{2} \sum_{i=1}^{L}\left(c_{i}^{\dagger} \mathrm{i}+1+\gamma c_{i}^{\dagger} c_{i+1}^{\dagger}\right)+\text { h.c. }+c_{i}^{\dagger} \dot{\mathrm{i}},
$$

where h.c. denotes the hermitian conjugate of the parenthesized expression in front. For $L$ large the second term can be neglected. For odd $L$ the second term vanishes. Thus by restriction to an odd number of particles, one can proceed the diagonalization procedure with the Hamiltonian

$$
H=\frac{L}{2}-\sum_{i=1}^{L} \frac{\lambda}{2}\left(c_{i}^{\dagger} \mathrm{i}+1+\gamma c_{i}^{\dagger} c_{i+1}^{\dagger}\right)+\text { h.c. }+c_{i}^{\dagger} \mathrm{i} .
$$

Next, one performs a Fourier transform to decouple the Hamiltonian into a direct product of Hilbert spaces preserving the fermionic anti-commutation relations. Let $\phi_{p}=2 \pi p / L$, then the operators i⿱ are given in the new operators $\underline{\mathrm{p}}$ as

$$
\mathrm{j}=\frac{1}{\sqrt{L}} \sum_{p=-L / 2}^{L / 2} \exp \left(-i j \phi_{p}\right) \underline{\mathrm{p}}
$$

where the summation runs over all possible momenta. Applying the Fourier transform (A13) to the Hamiltonian (A12) results in

$$
H=\frac{L}{2}-(\lambda+1) b_{0}^{\dagger} \underline{0}-\sum_{p=1}^{L / 2}\left(\lambda \cos \phi_{p}+1\right)\left(b_{p}^{\dagger} \underline{\mathrm{p}}+b_{-p}^{\dagger}-\underline{\mathrm{p}}\right)-i \gamma \lambda \sin \phi_{p}\left(b_{p}^{\dagger} b_{-p}^{\dagger}+\underline{\mathrm{p}}-\underline{\mathrm{p}}\right) .
$$

The Hilbert space decomposes into non-interacting subspaces. The space of zero momentum is two dimensional and already diagonal, whereas the other subspaces are four dimensional and non diagonal.

In order to diagonalize the remaining subspaces in the Hamiltonian (A14) let $\alpha_{p}=\left(\lambda \cos \phi_{p}+1\right), \beta_{p}=\lambda \gamma \sin \phi_{p}$ and use then the canonically transformed operators

$$
\eta_{k}=\tilde{\alpha}_{k}-\underline{\mathrm{k}}-i \tilde{\beta}_{k} b_{k}^{\dagger}
$$

with

$$
\begin{aligned}
\tilde{\alpha}_{k} & =\frac{\Lambda_{k}-\alpha_{k}}{\sqrt{2\left(\Lambda_{k}^{2}-\Lambda_{k} \alpha_{k}\right)}}, \\
\tilde{\beta}_{k} & =\frac{\beta_{k}}{\sqrt{2\left(\Lambda_{k}^{2}-\Lambda_{k} \alpha_{k}\right)}} \text { and } \\
\Lambda_{k} & =\sqrt{\alpha_{k}^{2}+\beta_{k}^{2}} .
\end{aligned}
$$

In the new creation and annihilation operators $\eta_{k}^{\dagger}$ and $\eta_{k}$ our Hamiltonian reads

$$
H=\sum_{k=-L / 2}^{L / 2} \Lambda_{k} \eta_{k}^{\dagger} \eta_{k}-\frac{1}{2} \sum_{k} \Lambda_{k}
$$

Clearly, the ground state of the system is given by the Fock vacuum in the fermionic basis. Although the vacuum state is separable in the fermionic basis this does not have to hold for the computational basis, since the Jordan-Wigner transformation is a global unitary transformation. To draw conclusions about the entanglement properties of the ground state one has to study the ground state in the computational basis.

\section{Expectation values of Pauli operators}

In order to calculate the three-particle [8] respectively four-particle reduced density matrices of the ground state $|0\rangle$ of our system one has to trace out all particles but three, respectively four. Alternatively one can choose a local 
operator basis on each system, which is not to be traced out and calculate the one-point up to four-point correlators to recover the reduced density matrix. For the reduced states on the tree particles $i, j$ and $k$ and the four particles $i$, $j, k$ and $l$ this would yield

$$
\begin{aligned}
\varrho_{i j k} & =\frac{1}{8} \sum_{m, n, o}\left\langle\sigma_{i}^{m} \sigma_{j}^{n} \sigma_{k}^{o}\right\rangle_{|0\rangle} \sigma_{i}^{m} \sigma_{j}^{n} \sigma_{k}^{o} \text { and } \\
\varrho_{i j k l} & =\frac{1}{16} \sum_{m, n, o, p}\left\langle\sigma_{i}^{m} \sigma_{j}^{n} \sigma_{k}^{o} \sigma_{l}^{p}\right\rangle_{|0\rangle} \sigma_{i}^{m} \sigma_{j}^{n} \sigma_{k}^{o} \sigma_{l}^{p},
\end{aligned}
$$

where each of the summations $m, n, o$ and $p$ run over $\{x, y, z, 0\}$. In order to calculate the expectation values $\left\langle\sigma_{i_{1}}^{\alpha_{1}} \ldots \sigma_{i_{k}}^{\alpha_{k}}\right\rangle_{|0\rangle}$ one needs to express the Pauli-operators in terms of the fermionic operators $\eta_{k}^{\dagger}$ and $\eta_{k}$. It is

$$
\begin{aligned}
& \sigma_{l}^{x}=\left(\mathrm{l}+c_{l}^{\dagger}\right) \prod_{i=1}^{l-1}\left(c_{i}^{\dagger}+\mathrm{i}\right)\left(c_{i}^{\dagger}-\mathrm{i}\right), \\
& \sigma_{l}^{z}=-\left(c_{l}^{\dagger}+\frac{1}{3}\right)\left(c_{l}^{\dagger}-\frac{1}{3}\right), \\
& \sigma_{l}^{y}=-i\left(c_{l}^{\dagger}-\frac{1}{3}\right) \prod_{i=1}^{l-1}\left(c_{i}^{\dagger}+\mathrm{i}\right)\left(c_{i}^{\dagger}-\mathrm{i}\right)
\end{aligned}
$$

and thus the Pauli operators are products of

$$
\begin{aligned}
& A_{l}=1+c_{l}^{\dagger}=\frac{1}{\sqrt{L}} \sum_{q}\left(\eta_{-q}^{\dagger}+\eta_{q}\right)\left(\alpha_{q}+i \beta_{q}\right) e^{i \phi_{q} l}, \\
& B_{l}=1-c_{l}^{\dagger}=\frac{1}{\sqrt{L}} \sum_{q}\left(-\eta_{-q}^{\dagger}+\eta_{q}\right)\left(\alpha_{q}-i \beta_{q}\right) e^{i \phi_{q} l},
\end{aligned}
$$

which are linear in the creation and annihilation operators $\eta_{i}^{\dagger}$ resp. $\eta_{i}$. The expectation values of arbitrary tensor products of

$$
\begin{aligned}
& \sigma_{l}^{x}=A_{l} \prod_{i=1}^{l-1} A_{i} B_{i}, \\
& \sigma_{l}^{z}=-A_{i} B_{i}, \\
& \sigma_{l}^{y}=-i B_{l} \prod_{i=1}^{l-1} A_{i} B_{i},
\end{aligned}
$$

are then monomials in the fermionic creation and annihilation operators. These can be evaluated using the Wick theorem, which states the equality

$$
\left\langle\mathcal{O}_{1} \cdots \mathcal{O}_{n}\right\rangle_{|0\rangle}=\left\langle\mathcal{O}_{1} \mathcal{O}_{2}\right\rangle_{|0\rangle}\left\langle\mathcal{O}_{3} \cdots \mathcal{O}_{n}\right\rangle_{|0\rangle}-\left\langle\mathcal{O}_{1} \mathcal{O}_{3}\right\rangle_{|0\rangle}\left\langle\mathcal{O}_{2} \mathcal{O}_{4} \cdots \mathcal{O}_{n}\right\rangle_{|0\rangle}+\left\langle\mathcal{O}_{1} \mathcal{O}_{4}\right\rangle_{|0\rangle} \cdots
$$

where $\mathcal{O}_{i}$ can be any operator $A_{j}$ or $B_{k}$. Successive application of the theorem reduces the expectation values in (A18) and its four-particle counterpart (A19) to products of the two-point expectation values $\left\langle A_{l} A_{k}\right\rangle_{|0\rangle},\left\langle A_{l} B_{k}\right\rangle_{|0\rangle}$ and $\left\langle B_{l} B_{k}\right\rangle_{|0\rangle}$. Using equations (A21) and (A22) and setting $r=k-l$ one can calculate these to be

$$
\begin{aligned}
& \left\langle A_{l} A_{k}\right\rangle_{|0\rangle}=\delta_{l k} \\
& \left\langle A_{l} B_{k}\right\rangle_{|0\rangle}=\frac{2}{\pi} \int_{0}^{\pi} d \phi(\cos \phi r(1+\lambda \cos \phi)-\gamma \lambda \sin \phi \sin \phi r) \frac{1}{\Lambda_{\phi}} \\
& \left\langle B_{l} B_{k}\right\rangle_{|0\rangle}=-\delta_{l k}
\end{aligned}
$$

in the thermodynamic limit. For finite $L$ equation (A26) is given by

$$
\left\langle A_{l} B_{k}\right\rangle_{|0\rangle}=\frac{1}{L} \sum_{q} \frac{1}{\Lambda_{q}}\left(\cos r \phi_{q}\left(1+\lambda \cos \phi_{q}\right)-\gamma \lambda \sin \phi_{q} \sin r \phi_{q}\right) .
$$


To evaluate $\left\langle\sigma_{i}^{m} \sigma_{j}^{n} \sigma_{k}^{o}\right\rangle_{|0\rangle}$ and $\left\langle\sigma_{i}^{m} \sigma_{j}^{n} \sigma_{k}^{o} \sigma_{l}^{p}\right\rangle_{|0\rangle}$ we express the Pauli operators in terms of $A_{j}$ or $B_{k}$. Then proceed with the general scheme by Ref. 28]. Reorder the operators with respect to the fermionic commutation relations, such that all $A_{l}$ are in front of the $B_{l}$ and both are in ascending order. After this step the expressions look like

$$
\pm\left\langle A_{i_{1}} \ldots A_{i_{k}} B_{j_{1}} \ldots B_{j_{k}}\right\rangle_{|0\rangle} .
$$

Applying the Wick theorem iteratively one obtains the following Pfaffian

$$
\pm p f\left[\begin{array}{ccccccc}
0 & \left\langle A_{i_{1}} A_{i_{2}}\right\rangle_{|0\rangle} & \cdots & \left\langle A_{i_{1}} A_{i_{k}}\right\rangle_{|0\rangle} & \left\langle A_{i_{1}} B_{j_{1}}\right\rangle_{|0\rangle} & \cdots & \left\langle A_{i_{1}} B_{j_{k}}\right\rangle_{|0\rangle} \\
-\left\langle A_{i_{1}} A_{i_{2}}\right\rangle_{|0\rangle} & 0 & & \left\langle A_{i_{2}} A_{i_{k}}\right\rangle_{|0\rangle} & \left\langle A_{i_{2}} B_{j_{1}}\right\rangle_{|0\rangle} & & \left\langle A_{i_{2}} B_{j_{k}}\right\rangle_{|0\rangle} \\
& & & \vdots & \vdots & & \vdots \\
& & \ddots & \left\langle A_{i_{k-1}} A_{i_{k}}\right\rangle_{|0\rangle} & \left\langle A_{i_{k-1}} B_{j_{1}}\right\rangle_{|0\rangle} & & \left\langle A_{i_{k-1}} B_{j_{k}}\right\rangle_{|0\rangle} \\
& & 0 & \left\langle A_{i_{k}} B_{j_{1}}\right\rangle_{|0\rangle} & \cdots & \left\langle A_{i_{k}} B_{j_{k}}\right\rangle_{|0\rangle} \\
& & & 0 & \cdots & \left\langle B_{j_{1}} B_{j_{k}}\right\rangle_{|0\rangle} \\
& & & & & \vdots \\
& & & & \ddots & \left\langle B_{j_{k-1}} B_{j_{k}}\right\rangle_{|0\rangle}
\end{array}\right] .
$$

Note that the upper left and the lower right $k \times k$ block of this Pfaffian are zero, since we have $i_{1}<i_{2}<\cdots<i_{k}$ and $j_{1}<j_{2}<\cdots<j_{k}$ together with (A25) and (A27). Such a Pfaffian can be expressed as

$$
p f\left[\begin{array}{cc}
0 & M \\
-M^{T} & 0
\end{array}\right]=(-1)^{\frac{k(k-1)}{2}} \operatorname{det} M
$$

and therefore we have

$$
\left\langle A_{i_{1}} \ldots A_{i_{k}} B_{j_{1}} \ldots B_{j_{k}}\right\rangle_{|0\rangle}=(-1)^{\frac{k(k-1)}{2}}\left|\begin{array}{ccc}
G_{j_{1}-i_{1}} & \ldots & G_{j_{k}-i_{1}} \\
\vdots & & \vdots \\
G_{j_{1}-i_{k}} & \ldots & G_{j_{k}-i_{k}}
\end{array}\right|
$$

where $G_{k-l}=\left\langle A_{l} B_{k}\right\rangle_{|0\rangle}$.

\section{Expectation values of four-site operator basis}

A list of all non-zero expectation values of the four-site Pauli operators $\left\langle\sigma_{i}^{m} \sigma_{j}^{n} \sigma_{k}^{o} \sigma_{l}^{p}\right\rangle_{|0\rangle}$ is presented here. The three site expectation values are omitted, since they are already contained in those of four sites. Note that there are 80 non-vanishing expectation values out of 256 . This is due to several symmetries the Hamiltonian possesses, and which carry over to its ground state. Namely the Hamiltonian is translationally invariant and thus just the spacings $\alpha=j-i, \beta=k-j$ and $\delta=k-l$ between the local sites enter the final expectation values. Furthermore, it is invariant under global $x$-flip $\sigma_{i}^{x} \rightarrow-\sigma_{i}^{x}$ for all $i$ and under global $y$-flip. Hence the only contribution to the four site Pauli-operators are those, in which $\sigma^{x}$ and $\sigma^{y}$ appear an even number of times. The exact numerical value finally depends on $\left\langle A_{l} B_{k}\right\rangle_{|0\rangle}=G_{k-l}$ only.

It is

$$
\begin{aligned}
& \langle\mathbb{1} \mathbb{1} \mathbb{1}\rangle_{|0\rangle}=1, \\
& \left\langle\sigma_{i}^{z} \mathbb{1} \mathbb{1} 1\right\rangle_{|0\rangle}=-G_{0}, \\
& \left\langle\sigma_{i}^{z} \sigma_{j}^{z} \mathbb{1} \mathbb{1}\right\rangle_{|0\rangle}=G_{0}^{2}-G_{\alpha} G_{-\alpha} .
\end{aligned}
$$

Similar it is $\left\langle\mathbb{1} \sigma_{j}^{z} \mathbb{1} \mathbb{1}\right\rangle_{|0\rangle}=\left\langle\mathbb{1} \mathbb{1} \sigma_{k}^{z} \mathbb{1}\right\rangle_{|0\rangle}=\left\langle\mathbb{1} \mathbb{1} 1 \sigma_{l}^{z}\right\rangle_{|0\rangle}=-G_{0}$. As for the tensor product of two $\sigma^{z}$ operators $\left\langle\sigma_{i}^{z} \mathbb{1} \sigma_{k}^{z} \mathbb{1}\right\rangle_{|0\rangle}=G_{0}^{2}-G_{\alpha+\beta} G_{-\alpha-\beta},\left\langle\sigma_{i}^{z} \mathbb{1} \mathbb{1} \sigma_{l}^{z}\right\rangle_{|0\rangle}=G_{0}^{2}-G_{\alpha+\beta+\delta} G_{-\alpha-\beta-\delta}, \quad\left\langle\mathbb{1} \sigma_{j}^{z} \sigma_{k}^{z} \mathbb{1}\right\rangle_{|0\rangle}=G_{0}^{2}-G_{\beta} G_{-\beta}$, $\left\langle\mathbb{1} \sigma_{j}^{z} \mathbb{1} \sigma_{j}^{z}\right\rangle_{|0\rangle}=G_{0}^{2}-G_{\beta+\delta} G_{-\beta-\delta},\left\langle\mathbb{1} \mathbb{1} \sigma_{k}^{z} \sigma_{l}^{z}\right\rangle_{|0\rangle}=G_{0}^{2}-G_{\delta} G_{-\delta}$.

$$
\left\langle\sigma_{i}^{z} \sigma_{j}^{z} \sigma_{k}^{z} \mathbb{1}\right\rangle_{|0\rangle}=-\left|\begin{array}{ccc}
G_{0} & G_{\alpha} & G_{\alpha+\beta} \\
G_{-\alpha} & G_{0} & G_{\beta} \\
G_{-\alpha-\beta} & G_{-\beta} & G_{0}
\end{array}\right|
$$


To get the expectation values of the other possible permutations of the above operator one simply has to perform the following replacements (applied from left to right) $\left\langle\sigma_{i}^{z} \sigma_{j}^{z} \mathbb{1} \sigma_{l}^{z}\right\rangle_{|0\rangle}: \beta \rightarrow \beta+\delta,\left\langle\sigma_{i}^{z} \mathbb{1} \sigma_{k}^{z} \sigma_{l}^{z}\right\rangle_{|0\rangle}: \beta \rightarrow \delta \wedge \alpha \rightarrow \alpha+\beta$ and $\left\langle\mathbb{1} \sigma_{j}^{z} \sigma_{k}^{z} \sigma_{l}^{z}\right\rangle_{|0\rangle}: \beta \rightarrow \delta \wedge \alpha \rightarrow \beta$.

$$
\begin{gathered}
\left\langle\sigma_{i}^{z} \sigma_{j}^{z} \sigma_{k}^{z} \sigma_{l}^{z}\right\rangle_{|0\rangle}=\left|\begin{array}{cccc}
G_{0} & G_{\alpha} & G_{\alpha+\beta} & G_{\alpha+\beta+\delta} \\
G_{-\alpha} & G_{0} & G_{\beta} & G_{\beta+\delta} \\
G_{-\alpha-\beta} & G_{-\beta} & G_{0} & G_{\delta} \\
G_{-\alpha-\beta-\delta} & G_{-\beta-\delta} & G_{-\delta} & G_{0}
\end{array}\right| . \\
\left\langle\sigma_{i}^{x} \sigma_{j}^{x} \mathbb{1} \mathbb{1}\right\rangle_{|0\rangle}=(-1)^{\alpha}\left|\begin{array}{ccc}
G_{-1} & \ldots & G_{\alpha-2} \\
\vdots & & \vdots \\
G_{-\alpha} & \ldots & G_{-1}
\end{array}\right|
\end{gathered}
$$

$\left\langle\sigma_{i}^{x} \mathbb{1} \sigma_{k}^{x} \mathbb{1}\right\rangle_{|0\rangle}: \alpha \rightarrow \alpha+\beta,\left\langle\sigma_{i}^{x} \mathbb{1} \mathbb{1} \sigma_{l}^{x}\right\rangle_{|0\rangle}: \alpha \rightarrow \alpha+\beta+\delta,\left\langle\mathbb{1} \sigma_{j}^{x} \sigma_{k}^{x} \mathbb{1}\right\rangle_{|0\rangle}: \alpha \rightarrow \beta,\left\langle\mathbb{1} \sigma_{j}^{x} \mathbb{1} \sigma_{l}^{x}\right\rangle_{|0\rangle}: \alpha \rightarrow \beta+\delta,\left\langle\mathbb{1} \mathbb{1} \sigma_{k}^{x} \sigma_{l}^{x}\right\rangle_{|0\rangle}:$ $\alpha \rightarrow \delta$.

$$
\left\langle\sigma_{i}^{x} \sigma_{j}^{x} \sigma_{k}^{z} \mathbb{1}\right\rangle_{|0\rangle}=(-1)^{\alpha+1}\left|\begin{array}{cccc}
G_{-1} & \ldots & G_{\alpha-2} & G_{\alpha+\beta-1} \\
\vdots & & \vdots & \vdots \\
G_{-\alpha} & \ldots & G_{-1} & G_{\beta} \\
G_{-\alpha-\beta} & \ldots & G_{-\beta-1} & G_{0}
\end{array}\right|,
$$

$\left\langle\sigma_{i}^{x} \sigma_{j}^{x} \mathbb{1} \sigma_{l}^{z}\right\rangle_{|0\rangle}: \beta \rightarrow \beta+\delta,\left\langle\sigma_{i}^{x} \mathbb{1} \sigma_{k}^{x} \sigma_{l}^{z}\right\rangle_{|0\rangle}: \beta \rightarrow \delta \wedge \alpha \rightarrow \alpha+\beta,\left\langle\mathbb{1} \sigma_{j}^{x} \sigma_{k}^{x} \sigma_{l}^{z}\right\rangle_{|0\rangle}: \beta \rightarrow \delta \wedge \alpha \rightarrow \beta$.

$$
\left\langle\sigma_{i}^{x} \sigma_{j}^{z} \sigma_{k}^{x} \mathbb{1}\right\rangle_{|0\rangle}=(-1)^{\alpha+\beta}\left|\begin{array}{cccccc}
G_{-1} & \ldots & G_{\alpha-2} & G_{\alpha} & \ldots & G_{\alpha+\beta-2} \\
\vdots & & \vdots & \vdots & & \vdots \\
G_{-\alpha+1} & \ldots & G_{0} & G_{2} & \ldots & G_{\beta} \\
G_{-\alpha-1} & \ldots & G_{-2} & G_{0} & \ldots & G_{\beta-2} \\
\vdots & & \vdots & \vdots & & \vdots \\
G_{-\alpha-\beta} & \ldots & G_{-\beta-1} & G_{-\beta+1} & \ldots & G_{-1}
\end{array}\right|
$$

$\left\langle\sigma_{i}^{x} \sigma_{j}^{z} \mathbb{1} \sigma_{l}^{x}\right\rangle_{|0\rangle}: \beta \rightarrow \beta+\delta,\left\langle\sigma_{i}^{x} \mathbb{1} \sigma_{k}^{z} \sigma_{l}^{x}\right\rangle_{|0\rangle}: \beta \rightarrow \delta \wedge \alpha \rightarrow \alpha+\beta,\left\langle\mathbb{1} \sigma_{j}^{x} \sigma_{k}^{z} \sigma_{l}^{x}\right\rangle_{|0\rangle}: \beta \rightarrow \delta \wedge \alpha \rightarrow \beta$.

$$
\left\langle\sigma_{i}^{z} \sigma_{j}^{x} \sigma_{k}^{x} \mathbb{1}\right\rangle_{|0\rangle}=(-1)^{\beta+1}\left|\begin{array}{cccc}
G_{0} & G_{\alpha} & \ldots & G_{\alpha+\beta-1} \\
G_{-\alpha-1} & G_{-1} & \ldots & G_{\beta-2} \\
\vdots & \vdots & & \vdots \\
G_{-\alpha-\beta} & G_{-\beta} & \ldots & G_{-1}
\end{array}\right|,
$$

$\left\langle\sigma_{i}^{z} \sigma_{j}^{x} \mathbb{1} \sigma_{l}^{x}\right\rangle_{|0\rangle}: \beta \rightarrow \beta+\delta,\left\langle\sigma_{i}^{z} \mathbb{1} \sigma_{k}^{x} \sigma_{l}^{x}\right\rangle_{|0\rangle}: \beta \rightarrow \delta \wedge \alpha \rightarrow \alpha+\beta,\left\langle\mathbb{1} \sigma_{j}^{z} \sigma_{k}^{x} \sigma_{l}^{x}\right\rangle_{|0\rangle}: \beta \rightarrow \delta \wedge \alpha \rightarrow \beta$.

$$
\left\langle\sigma_{i}^{y} \sigma_{j}^{y} \mathbb{1} \mathbb{1}\right\rangle_{|0\rangle}=(-1)^{\alpha}\left|\begin{array}{ccc}
G_{1} & \ldots & G_{\alpha} \\
\vdots & & \vdots \\
G_{-\alpha+2} & \ldots & G_{1}
\end{array}\right|
$$

$\left\langle\sigma_{i}^{y} \mathbb{1} \sigma_{k}^{y} \mathbb{1}\right\rangle_{|0\rangle}: \alpha \rightarrow \alpha+\beta,\left\langle\sigma_{i}^{y} \mathbb{1} \mathbb{1} \sigma_{l}^{y}\right\rangle_{|0\rangle}: \alpha \rightarrow \alpha+\beta+\delta,\left\langle\mathbb{1} \sigma_{j}^{y} \sigma_{k}^{y} \mathbb{1}\right\rangle_{|0\rangle}: \alpha \rightarrow \beta,\left\langle\mathbb{1} \sigma_{j}^{y} \mathbb{1} \sigma_{l}^{y}\right\rangle_{|0\rangle}: \alpha \rightarrow \beta+\delta,\left\langle\mathbb{1} \mathbb{1} \sigma_{k}^{y} \sigma_{l}^{y}\right\rangle_{|0\rangle}:$ $\alpha \rightarrow \delta$.

$$
\left\langle\sigma_{i}^{y} \sigma_{j}^{y} \sigma_{k}^{z} \mathbb{1}\right\rangle_{|0\rangle}=(-1)^{\alpha+1}\left|\begin{array}{cccc}
G_{1} & \ldots & G_{\alpha} & G_{\alpha+\beta} \\
\vdots & & \vdots & \vdots \\
G_{-\alpha+2} & \ldots & G_{1} & G_{\beta+1} \\
G_{-\alpha-\beta+1} & \ldots & G_{-\beta} & G_{0}
\end{array}\right|
$$


$\left\langle\sigma_{i}^{y} \sigma_{j}^{y} \mathbb{1} \sigma_{l}^{z}\right\rangle_{|0\rangle}: \beta \rightarrow \beta+\delta,\left\langle\sigma_{i}^{y} \mathbb{1} \sigma_{k}^{y} \sigma_{l}^{z}\right\rangle_{|0\rangle}: \beta \rightarrow \delta \wedge \alpha \rightarrow \alpha+\beta,\left\langle\mathbb{1} \sigma_{j}^{y} \sigma_{k}^{y} \sigma_{l}^{z}\right\rangle_{|0\rangle}: \beta \rightarrow \delta \wedge \alpha \rightarrow \beta$

$$
\left\langle\sigma_{i}^{y} \sigma_{j}^{z} \sigma_{k}^{y} \mathbb{1}\right\rangle_{|0\rangle}=(-1)^{\alpha+\beta}\left|\begin{array}{cccccc}
G_{1} & \ldots & G_{\alpha-1} & G_{\alpha+1} & \ldots & G_{\alpha+\beta} \\
\vdots & & \vdots & \vdots & & \vdots \\
G_{-\alpha+2} & \ldots & G_{0} & G_{2} & \ldots & G_{\beta+1} \\
G_{-\alpha} & \ldots & G_{-2} & G_{0} & \ldots & G_{\beta-1} \\
\vdots & & \vdots & \vdots & & \vdots \\
G_{-\alpha-\beta+2} & \ldots & G_{-\beta} & G_{-\beta+2} & \ldots & G_{1}
\end{array}\right|,
$$

$\left\langle\sigma_{i}^{y} \sigma_{j}^{z} \mathbb{1} \sigma_{l}^{y}\right\rangle_{|0\rangle}: \beta \rightarrow \beta+\delta,\left\langle\sigma_{i}^{y} \mathbb{1} \sigma_{k}^{z} \sigma_{l}^{y}\right\rangle_{|0\rangle}: \beta \rightarrow \delta \wedge \alpha \rightarrow \alpha+\beta,\left\langle\mathbb{1} \sigma_{j}^{y} \sigma_{k}^{z} \sigma_{l}^{y}\right\rangle_{|0\rangle}: \beta \rightarrow \delta \wedge \alpha \rightarrow \beta$.

$$
\left\langle\sigma_{i}^{z} \sigma_{j}^{y} \sigma_{k}^{y} \mathbb{1}\right\rangle_{|0\rangle}=(-1)^{\beta+1}\left|\begin{array}{cccc}
G_{0} & G_{\alpha+1} & \ldots & G_{\alpha+\beta} \\
G_{-\alpha} & G_{1} & \ldots & G_{\beta} \\
\vdots & \vdots & & \vdots \\
G_{-\alpha-\beta+1} & G_{-\beta+2} & \ldots & G_{1}
\end{array}\right|,
$$

$\left\langle\sigma_{i}^{z} \sigma_{j}^{y} \mathbb{1} \sigma_{l}^{y}\right\rangle_{|0\rangle}: \beta \rightarrow \beta+\delta,\left\langle\sigma_{i}^{z} \mathbb{1} \sigma_{k}^{y} \sigma_{l}^{y}\right\rangle_{|0\rangle}: \beta \rightarrow \delta \wedge \alpha \rightarrow \alpha+\beta,\left\langle\mathbb{1} \sigma_{j}^{z} \sigma_{k}^{y} \sigma_{l}^{y}\right\rangle_{|0\rangle}: \beta \rightarrow \delta \wedge \alpha \rightarrow \beta$.

$$
\begin{aligned}
& \left\langle\sigma_{i}^{x} \sigma_{j}^{x} \sigma_{k}^{z} \sigma_{l}^{z}\right\rangle_{|0\rangle}=(-1)^{\alpha}\left|\begin{array}{ccccc}
G_{-1} & \ldots & G_{\alpha-2} & G_{\alpha+\beta-1} & G_{\alpha+\beta+\delta-1} \\
\vdots & & \vdots & \vdots & \vdots \\
G_{-\alpha} & \ldots & G_{-1} & G_{\beta} & G_{\beta+\delta} \\
G_{-\alpha-\beta} & \ldots & G_{-\beta-1} & G_{0} & G_{\delta} \\
G_{-\alpha-\beta-\delta} & \ldots & G_{-\beta-\delta-1} & G_{-\delta} & G_{0}
\end{array}\right| \\
& \left\langle\sigma_{i}^{z} \sigma_{j}^{z} \sigma_{k}^{x} \sigma_{l}^{x}\right\rangle_{|0\rangle}=(-1)^{\delta}\left|\begin{array}{ccccc}
G_{0} & G_{\alpha} & G_{\alpha+\beta} & \ldots & G_{\alpha+\beta+\delta-1} \\
G_{-\alpha} & G_{0} & G_{\beta} & \ldots & G_{\beta+\delta-1} \\
G_{-\alpha-\beta-1} & G_{-\beta-1} & G_{-1} & \ldots & G_{\delta-2} \\
\vdots & \vdots & \vdots & & \vdots \\
G_{-\alpha-\beta-\delta} & G_{-\beta-\delta} & G_{-\delta} & \ldots & G_{-1}
\end{array}\right| \\
& \left\langle\sigma_{i}^{z} \sigma_{j}^{x} \sigma_{k}^{x} \sigma_{l}^{z}\right\rangle_{|0\rangle}=(-1)^{\beta}\left|\begin{array}{ccccc}
G_{0} & G_{\alpha} & \ldots & G_{\alpha+\beta-1} & G_{\alpha+\beta+\delta} \\
G_{-\alpha-1} & G_{-1} & \ldots & G_{\beta-2} & G_{\beta+\delta-1} \\
\vdots & \vdots & & \vdots & \vdots \\
G_{-\alpha-\beta} & G_{-\beta} & \ldots & G_{-1} & G_{\delta} \\
G_{-\alpha-\beta-\delta} & G_{-\beta-\delta} & \ldots & G_{-\delta-1} & G_{0}
\end{array}\right| \\
& \left\langle\sigma_{i}^{x} \sigma_{j}^{z} \sigma_{k}^{z} \sigma_{l}^{x}\right\rangle_{|0\rangle}=(-1)^{\alpha+\beta+\delta}\left|\begin{array}{cccccccccc}
G_{-1} & \ldots & G_{\alpha-2} & G_{\alpha} & \ldots & G_{\alpha+\beta-2} & G_{\alpha+\beta} & \ldots & G_{\alpha+\beta+\delta-2} \\
\vdots & & \vdots & \vdots & & \vdots & \vdots & & \vdots \\
G_{-\alpha+1} & \ldots & G_{0} & G_{2} & \ldots & G_{\beta} & G_{\beta+2} & \ldots & G_{\beta+\delta} \\
G_{-\alpha-1} & \ldots & G_{-2} & G_{0} & \ldots & G_{\beta-2} & G_{\beta} & \ldots & G_{\beta+\delta-2} \\
\vdots & & \vdots & \vdots & & \vdots & \vdots & & \vdots \\
G_{-\alpha-\beta+1} & \ldots & G_{-\beta} & G_{-\beta+2} & \ldots & G_{0} & G_{2} & \ldots & G_{\delta} \\
G_{-\alpha-\beta-1} & \ldots & G_{-\beta-2} & G_{-\beta} & \ldots & G_{-2} & G_{0} & \ldots & G_{\delta-2} \\
\vdots & & \vdots & \vdots & & \vdots & \vdots & & \vdots \\
G_{-\alpha-\beta-\delta} & \ldots & G_{-\beta-\delta-1} & G_{-\beta-\delta+1} & \ldots & G_{-\delta-1} & G_{-\delta+1} & \ldots & G_{-1}
\end{array}\right| \\
& \left\langle\sigma_{i}^{y} \sigma_{j}^{y} \sigma_{k}^{z} \sigma_{l}^{z}\right\rangle_{|0\rangle}=(-1)^{\alpha}\left|\begin{array}{ccccc}
G_{1} & \ldots & G_{\alpha} & G_{\alpha+\beta} & G_{\alpha+\beta+\delta} \\
\vdots & & \vdots & \vdots & \vdots \\
G_{-\alpha+2} & \ldots & G_{1} & G_{\beta+1} & G_{\beta+\delta+1} \\
G_{-\alpha-\beta+1} & \ldots & G_{-\beta} & G_{0} & G_{\delta} \\
G_{-\alpha-\beta-\delta+1} & \ldots & G_{-\beta-\delta} & G_{-\delta} & G_{0}
\end{array}\right|
\end{aligned}
$$




$$
\begin{aligned}
& \left\langle\sigma_{i}^{z} \sigma_{j}^{z} \sigma_{k}^{y} \sigma_{l}^{y}\right\rangle_{|0\rangle}=(-1)^{\delta}\left|\begin{array}{ccccc}
G_{0} & G_{\alpha} & G_{\alpha+\beta+1} & \ldots & G_{\alpha+\beta+\delta} \\
G_{-\alpha} & G_{0} & G_{\beta+1} & \ldots & G_{\beta+\delta} \\
G_{-\alpha-\beta} & G_{-\beta} & G_{1} & \ldots & G_{\delta} \\
\vdots & \vdots & \vdots & & \vdots \\
G_{-\alpha-\beta-\delta+1} & G_{-\beta-\delta+1} & G_{-\delta+2} & \ldots & G_{1}
\end{array}\right| \\
& \left\langle\sigma_{i}^{z} \sigma_{j}^{y} \sigma_{k}^{y} \sigma_{l}^{z}\right\rangle_{|0\rangle}=(-1)^{\beta}\left|\begin{array}{ccccc}
G_{0} & G_{\alpha+1} & \ldots & G_{\alpha+\beta} & G_{\alpha+\beta+\delta} \\
G_{-\alpha} & G_{1} & \ldots & G_{\beta} & G_{\beta+\delta} \\
\vdots & \vdots & & \vdots & \vdots \\
G_{-\alpha-\beta+1} & G_{-\beta+2} & \ldots & G_{1} & G_{\delta+1} \\
G_{-\alpha-\beta-\delta} & G_{-\beta-\delta+1} & \ldots & G_{-\delta} & G_{0}
\end{array}\right| \\
& \begin{array}{c|ccccccccc}
G_{1} & \ldots & G_{\alpha-1} & G_{\alpha+1} & \ldots & G_{\alpha+\beta-1} & G_{\alpha+\beta+1} & \ldots & G_{\alpha+\beta+\delta} \\
\vdots & & \vdots & \vdots & & \vdots & \vdots & & \vdots \\
G_{-\alpha+2} & \ldots & G_{0} & G_{2} & \ldots & G_{\beta} & G_{\beta+2} & \ldots & G_{\beta+\delta+1} \\
\left.G_{-\alpha} \sigma_{j}^{z} \sigma_{k}^{z} \sigma_{l}^{y}\right\rangle_{|0\rangle}=(-1)^{\alpha+\beta+\delta} & \ldots & G_{-2} & G_{0} & \ldots & G_{\beta-2} & G_{\beta} & \ldots & G_{\beta+\delta-1} \\
\vdots & & \vdots & \vdots & & \vdots & \vdots & & \vdots \\
G_{-\alpha-\beta+2} & \ldots & G_{-\beta} & G_{-\beta+2} & \ldots & G_{0} & G_{2} & \ldots & G_{\delta+1} \\
G_{-\alpha-\beta} & \ldots & G_{-\beta-2} & G_{-\beta} & \ldots & G_{-2} & G_{0} & \ldots & G_{\delta-1} \\
\vdots & & \vdots & \vdots & & \vdots & \vdots & & \vdots \\
G_{-\alpha-\beta-\delta+2} & \ldots & G_{-\beta-\delta} & G_{-\beta-\delta+2} & \ldots & G_{-\delta} & G_{-\delta+2} & \ldots & G_{1}
\end{array} \mid \\
& \left\langle\sigma_{i}^{x} \sigma_{j}^{z} \sigma_{k}^{x} \sigma_{l}^{z}\right\rangle_{|0\rangle}=(-1)^{\alpha+\beta+1}\left|\begin{array}{ccccccc}
G_{-1} & \ldots & G_{\alpha-2} & G_{\alpha} & \ldots & G_{\alpha+\beta-2} & G_{\alpha+\beta+\delta-1} \\
\vdots & & \vdots & \vdots & & \vdots & \vdots \\
G_{-\alpha+1} & \ldots & G_{0} & G_{2} & \ldots & G_{\beta} & G_{\beta+\delta+1} \\
G_{-\alpha-1} & \ldots & G_{-2} & G_{0} & \ldots & G_{\beta-2} & G_{\beta+\delta-1} \\
\vdots & & \vdots & \vdots & & \vdots & \vdots \\
G_{-\alpha-\beta} & \ldots & G_{-\beta-1} & G_{-\beta+1} & \ldots & G_{-1} & G_{\delta} \\
G_{-\alpha-\beta-\delta} & \ldots & G_{-\beta-\delta-1} & G_{-\beta-\delta+1} & \ldots & G_{-\delta-1} & G_{0}
\end{array}\right| \\
& \left\langle\sigma_{i}^{z} \sigma_{j}^{x} \sigma_{k}^{z} \sigma_{l}^{x}\right\rangle_{|0\rangle}=(-1)^{\beta+\delta+1}\left|\begin{array}{ccccccc}
G_{0} & G_{\alpha} & \ldots & G_{\alpha+\beta-1} & G_{\alpha+\beta+1} & \ldots & G_{\alpha+\beta+\delta-1} \\
G_{-\alpha-1} & G_{-1} & \ldots & G_{\beta-2} & G_{\beta} & \ldots & G_{\beta+\delta-2} \\
\vdots & \vdots & & \vdots & \vdots & & \vdots \\
G_{-\alpha-\beta+1} & G_{-\beta+1} & \ldots & G_{0} & G_{2} & \ldots & G_{\delta} \\
G_{-\alpha-\beta-1} & G_{-\beta-1} & \ldots & G_{-2} & G_{0} & \ldots & G_{\delta-2} \\
\vdots & \vdots & & \vdots & \vdots & & \vdots \\
G_{-\alpha-\beta-\delta} & G_{-\beta-\delta} & \ldots & G_{-\delta-1} & G_{-\delta+1} & \ldots & G_{-1}
\end{array}\right| \\
& \left\langle\sigma_{i}^{y} \sigma_{j}^{z} \sigma_{k}^{y} \sigma_{l}^{z}\right\rangle_{|0\rangle}=(-1)^{\alpha+\beta+1}\left|\begin{array}{ccccccc}
G_{1} & \ldots & G_{\alpha-1} & G_{\alpha+1} & \ldots & G_{\alpha+\beta} & G_{\alpha+\beta+\delta} \\
\vdots & & \vdots & \vdots & & \vdots & \vdots \\
G_{-\alpha+2} & \ldots & G_{0} & G_{2} & \ldots & G_{\beta+1} & G_{\beta+\delta+1} \\
G_{-\alpha} & \ldots & G_{-2} & G_{0} & \ldots & G_{\beta-1} & G_{\beta+\delta-1} \\
\vdots & & \vdots & \vdots & & \vdots & \vdots \\
G_{-\alpha-\beta+2} & \ldots & G_{-\beta} & G_{-\beta+2} & \ldots & G_{1} & G_{\delta+1} \\
G_{-\alpha-\beta-\delta+1} & \ldots & G_{-\beta-\delta-1} & G_{-\beta-\delta+1} & \ldots & G_{-\delta} & G_{0}
\end{array}\right|
\end{aligned}
$$




$$
\left\langle\sigma_{i}^{z} \sigma_{j}^{y} \sigma_{k}^{z} \sigma_{l}^{y}\right\rangle_{|0\rangle}=(-1)^{\beta+\delta+1}\left|\begin{array}{ccccccc}
G_{0} & G_{\alpha+1} & \ldots & G_{\alpha+\beta-1} & G_{\alpha+\beta+1} & \ldots & G_{\alpha+\beta+\delta} \\
G_{-\alpha} & G_{1} & \ldots & G_{\beta-1} & G_{\beta+1} & \ldots & G_{\beta+\delta} \\
\vdots & \vdots & & \vdots & \vdots & & \vdots \\
G_{-\alpha-\beta+1} & G_{-\beta+2} & \ldots & G_{0} & G_{2} & \ldots & G_{\delta+1} \\
G_{-\alpha-\beta-1} & G_{-\beta} & \ldots & G_{-2} & G_{0} & \ldots & G_{\delta-1} \\
\vdots & \vdots & & \vdots & \vdots & & \vdots \\
G_{-\alpha-\beta-\delta+1} & G_{-\beta-\delta-2} & \ldots & G_{-\delta} & G_{-\delta+2} & \ldots & G_{1}
\end{array}\right|
$$

$$
\left\langle\sigma_{i}^{x} \sigma_{j}^{x} \sigma_{k}^{y} \sigma_{l}^{y}\right\rangle_{|0\rangle}=(-1)^{\alpha+\delta}\left|\begin{array}{cccccc}
G_{-1} & \ldots & G_{\alpha-2} & G_{\alpha+\beta} & \ldots & G_{\alpha+\beta+\delta-1} \\
\vdots & & \vdots & \vdots & & \vdots \\
G_{-\alpha} & \ldots & G_{-1} & G_{\beta+1} & \ldots & G_{\beta+\delta} \\
G_{-\alpha-\beta} & \ldots & G_{-\beta-1} & G_{1} & \ldots & G_{\delta} \\
\vdots & & \vdots & \vdots & & \vdots \\
G_{-\alpha-\beta-\delta+1} & \ldots & G_{-\beta-\delta} & G_{-\delta+2} & \ldots & G_{1}
\end{array}\right|
$$

$$
\left\langle\sigma_{i}^{y} \sigma_{j}^{y} \sigma_{k}^{x} \sigma_{l}^{x}\right\rangle_{|0\rangle}=(-1)^{\alpha+\delta}\left|\begin{array}{cccccc}
G_{1} & \ldots & G_{\alpha} & G_{\alpha+\beta} & \ldots & G_{\alpha+\beta+\delta-1} \\
\vdots & & \vdots & \vdots & & \vdots \\
G_{-\alpha+2} & \ldots & G_{1} & G_{\beta+1} & \ldots & G_{\beta+\delta} \\
G_{-\alpha-\beta} & \ldots & G_{-\beta-1} & G_{-1} & \ldots & G_{\delta-2} \\
\vdots & & \vdots & \vdots & & \vdots \\
G_{-\alpha-\beta-\delta+1} & \ldots & G_{-\beta-\delta} & G_{-\delta} & \ldots & G_{-1}
\end{array}\right|
$$

$$
\left\langle\sigma_{i}^{x} \sigma_{j}^{y} \sigma_{k}^{y} \sigma_{l}^{x}\right\rangle_{|0\rangle}=(-1)^{\alpha+\delta}\left|\begin{array}{cccccc}
G_{-1} & \ldots & G_{\alpha-1} & G_{\alpha+\beta} & \ldots & G_{\alpha+\beta+\delta-2} \\
\vdots & & \vdots & \vdots & & \vdots \\
G_{-\alpha+1} & \ldots & G_{1} & G_{\beta+2} & \ldots & G_{\beta+\delta} \\
G_{-\alpha-\beta} & \ldots & G_{-\beta} & G_{1} & \ldots & G_{\delta-1} \\
\vdots & & \vdots & \vdots & & \vdots \\
G_{-\alpha-\beta-\delta} & \ldots & G_{-\beta-\delta} & G_{-\delta+1} & \ldots & G_{-1}
\end{array}\right|
$$

$$
\left\langle\sigma_{i}^{y} \sigma_{j}^{x} \sigma_{k}^{x} \sigma_{l}^{y}\right\rangle_{|0\rangle}=(-1)^{\alpha+\delta}\left|\begin{array}{cccccc}
G_{1} & \ldots & G_{\alpha-1} & G_{\alpha+\beta} & \ldots & G_{\alpha+\beta+\delta} \\
\vdots & & \vdots & \vdots & & \vdots \\
G_{-\alpha+1} & \ldots & G_{-1} & G_{\beta} & \ldots & G_{\beta+\delta} \\
G_{-\alpha-\beta} & \ldots & G_{-\beta-2} & G_{-1} & \ldots & G_{\delta-1} \\
\vdots & & \vdots & \vdots & & \vdots \\
G_{-\alpha-\beta-\delta+2} & \ldots & G_{-\beta-\delta} & G_{-\delta+1} & \ldots & G_{1}
\end{array}\right|
$$

$$
\left\langle\sigma_{i}^{x} \sigma_{j}^{x} \sigma_{k}^{x} \sigma_{l}^{x}\right\rangle_{|0\rangle}=(-1)^{\alpha+\delta}\left|\begin{array}{cccccc}
G_{-1} & \ldots & G_{\alpha-2} & G_{\alpha+\beta-1} & \ldots & G_{\alpha+\beta+\delta-2} \\
\vdots & & \vdots & \vdots & & \vdots \\
G_{-\alpha} & \ldots & G_{-1} & G_{\beta} & \ldots & G_{\beta+\delta-1} \\
G_{-\alpha-\beta-1} & \ldots & G_{-\beta-2} & G_{-1} & \ldots & G_{\delta-2} \\
\vdots & & \vdots & \vdots & & \vdots \\
G_{-\alpha-\beta-\delta} & \ldots & G_{-\beta-\delta-1} & G_{-\delta} & \ldots & G_{-1}
\end{array}\right|
$$




$$
\left\langle\sigma_{i}^{y} \sigma_{j}^{y} \sigma_{k}^{y} \sigma_{l}^{y}\right\rangle_{|0\rangle}=(-1)^{\alpha+\delta}\left|\begin{array}{cccccc}
G_{1} & \ldots & G_{\alpha} & G_{\alpha+\beta+1} & \ldots & G_{\alpha+\beta+\delta} \\
\vdots & & \vdots & \vdots & & \vdots \\
G_{-\alpha+2} & \ldots & G_{1} & G_{\beta+2} & \ldots & G_{\beta+\delta+1} \\
G_{-\alpha-\beta+1} & \ldots & G_{-\beta} & G_{1} & \ldots & G_{\delta} \\
\vdots & & \vdots & \vdots & & \vdots \\
G_{-\alpha-\beta-\delta+2} & \ldots & G_{-\beta-\delta+1} & G_{-\delta+2} & \ldots & G_{1}
\end{array}\right|
$$

[1] A. Osterloh, L. Amico, G. Falci and R. Fazio, Nature 416, 608 (2002).

[2] T. J. Osborne and M. A. Nielsen, Phys. Rev. A 66032110 (2002).

[3] G. Vidal, J.I. Latorre, E. Rico, A. Kitaev, Phys. Rev. Lett. 90, 227902 (2003).

[4] L. A. Wu, M. S. Sarandy, and D. A. Lidar, Phys. Rev. Lett. 93, 250404 (2004).

[5] T. Roscilde, P. Verrucchi, A. Fubini, S. Haas, and V. Tognetti, Phys. Rev. Lett. 93, 167203 (2004).

[6] For recent results see P. Nataf, M. Dogan, and K. Le Hur, Phys. Rev. A 86, 043807 (2012); Y. Yao, H.-W. Li, Ch.-M. Zhang, Zh.-Q. Yin, W. Chen, G.- C. Guo, and Zh.-F. Han, Phys. Rev. A 86, 042102 (2012); F. Altintas and R. Eryigit, Ann. Phys. (USA) 3273084 (2012); L. Lepori, G. De Chiara, and A. Sanpera, Phys. Rev. B 87, 235107 (2013); V. Azimi Mousolou, C. M. Canali, and E. Sjöqvist, Phys. Rev. A 88, 012310 (2013); for a review see L. Amico, R. Fazio, A. Osterloh, and V. Vedral, Rev. Mod. Phys. 80, 517 (2008).

[7] For reviews see F. Verstraete, J.I. Cirac, V. Murg, Adv. Phys. 57, 143 (2008); N. Schuch, arXiv:1306.5551.

[8] D. Patanè, R. Fazio and L. Amico, New J. Phys. 9322 (2007).

[9] S. M. Giampaolo and B.C. Hiesmayr, Phys. Rev. A 88, 052305 (2013).

[10] J. Stasińska, B. Rogers, M. Paternostro, G. De Chiara, and A. Sanpera, Phys. Rev. A 89, 032330 (2014).

[11] T.-C. Wei, D. Das, S. Mukhopadyay, S. Vishveshwara, and P.M. Goldbart, Phys. Rev. A 71, 060305(R) (2005); O. Gühne, G. Tóth, and H.J. Briegel, New J. Phys. 7, 229 (2005); R. Orús, Phys. Rev. Lett. 100, 130502 (2008); H. S. Dhar, A. Sen(De), and U. Sen, Phys. Rev. Lett. 111, 070501 (2013); H. S. Dhar, A. Sen(De), and U. Sen, New J. Phys. 15, 013043 (2013).

[12] J. Batle and M. Casas, Phys. Rev. A 82, 062101 (2010).

[13] M. Srednicki, Phys. Rev. Lett. 71, 666 (1993); C. Holzhey, F. Larsen and F. Wilczek, Nucl. Phys. B 424, 44 (1994); V.E. Korepin, Phys. Rev. Lett. 92, 096402 (2004); M.B. Hastings, J. Stat. Mech. P08024 (2007); G. Vitagliano, A. Riera, and J. I. Latorre, New J. Phys. 12113049 (2010); P. Calabrese, M. Mintchev, E. Vicari, Eur. Phys. Lett. 9720009 (2012); P. Zanardi and L. C. Venuti, J. Stat. Mech. P04023 (2013); J. Haegeman, T. J. Osborne, H. Verschelde, and F. Verstraete, Phys. Rev. Lett. 110, 100402 (2013); B. Collins, I. Nechita, and K. Zyczkowski, J. Phys. A: Math. Theor. 46, 305302 (2013).

[14] J. Eisert, M. Cramer, M.B. Plenio, Rev. Mod. Phys. 82, 277 (2010).
[15] B. Jungnitsch, T. Moroder and O. Gühne, Phys. Rev. Lett. 106, 190502 (2011).

[16] J. T. Barreiro, P. Schindler, O. Gühne, T. Monz, M. Chwalla, C. F. Roos, M. Hennrich, and R. Blatt, Nature Phys. 6, 943 (2010); H. Kampermann, O. Gühne, C. Wilmott, and D. Bruß, Phys. Rev. A 86, 032307 (2012).

[17] E. Lieb, T. Schultz and D. Mattis, Ann. Phys. (N.Y.) 16 , 407 (1961); P. Pfeuty, Ann. Phys. (N.Y.) 57, 79 (1970); E. Barouch, B.M. McCoy and M. Dresden, Phys. Rev. A 2, 1075 (1970); E. Barouch, B.M. McCoy and M. Barry, Phys. Rev. A 3, 786 (1971).

[18] A. Osterloh and J. Siewert, Phys. Rev. A 72, 012337 (2005).

[19] O. Gühne and G. Tóth, Phys. Rep. 474, 1 (2009).

[20] A. Peres, Phys. Rev. Lett. 77, 1413 (1996).

[21] O. Gühne, B. Jungnitsch, T. Moroder, and Y.S. Weinstein, Phys. Rev. A 84, 052319 (2011); L. Novo, T. Moroder, and O. Gühne, Phys. Rev. A 88, 012305 (2013).

[22] M. E. Fisher, in "Fenomeni critici" (ed. by. M.S. Green), Academic Press (New York), 1971.

[23] M.N. Barber, in "Phase transitions and critical phenomena" (ed. by C. Domb, J.L. Lebowitz), Academic Press (New York), 1983.

[24] A. Uhlmann, Phys. Rev. A 62, 032307 (2000).

[25] A. Sen(De), U. Sen, and M. Lewenstein, Phys. Rev. A 72, 052319 (2005).

[26] See the program PPTmixer, available at mathworks. com/matlabcentral/fileexchange/30968.

[27] L. Gurvits and H. Barnum, Phys. Rev. A 66, 062311 (2002).

[28] E.R. Caianiello and S. Fubini, Nuovo Cimento 91218 (1953).

[29] Sometimes the notion of "genuine multiparticle entanglement" is used in a more strict way, by requiring in addition that the state has a non-vanishing value of some globally $S L$-invariant quantity [18]. These states are discerned by states in the null-cone of global $S L$-invariance.

[30] Formally, the partial transposition of a bipartite state $\varrho=\sum_{i j, k l} \varrho_{i j, k l}|i\rangle\langle j|\otimes| k\rangle\langle l|$ with respect to the second system is defined as $\varrho^{T_{B}}=\sum_{i j, k l} \varrho_{i j, k l}|i\rangle\langle j|\otimes| l\rangle\langle k|$.

[31] In many systems the shift exponent $\kappa$ equals the inverse of critical exponent $\nu$ of the diverging correlation length [23]. In our case, however, this turns out to be not the case.

[32] But note that the argument of Ref. [4] in the strict sense concerns only entanglement in $k$-particle marginals of a ground state of an $k$-body Hamiltonian. 